\title{
CHEMICAL EVOLUTION OF GALAXIES - CHALLENGES FOR STELLAR ASTRONOMY
}

\author{
ELAINE M. SADLER \\ School of Physics, University of Sydney \\ NSW 2006, Australia
}

\begin{abstract}
I discuss several current problems in understanding the chemical evolution of galaxies which have particular relevance for fundamental stellar astronomy.
\end{abstract}

\section{Introduction}

Stellar astronomy is the foundation on which we build our understanding of the evolution of galaxies and their stellar populations. A new generation of ground-based and space telescopes are now providing data of unprecedented quality for distant galaxies, so that it is becoming possible to investigate the stellar populations of these galaxies from spectroscopic studies of their integrated light (e.g. O'Connell 1995; Steidel et al. 1996).

Here, I will discuss three current problems in the chemical evolution of galaxies which relate closely to fundamental stellar astronomy. They are:

- Hot, UV-bright stars in old, metal-rich populations

- $\alpha$-element abundances, $\mathrm{Mg} / \mathrm{Fe}$ and the "iron discrepancy"

- Dating elliptical galaxies, and the age-metallicity relation

There is a unifying theme to this discussion, which is reflected in the assertion below.

The Galactic bulge is the 'new frontier' for stellar astronomy. It is being opened up by a new generation of large telescopes such as Keck and the ESO VLT, which will allow old, metal-rich stars to be studied in the kind of detail previously reserved for solar-neighbourhood disk stars. Such studies will also help improve our understanding of the evolution of distant galaxies. 


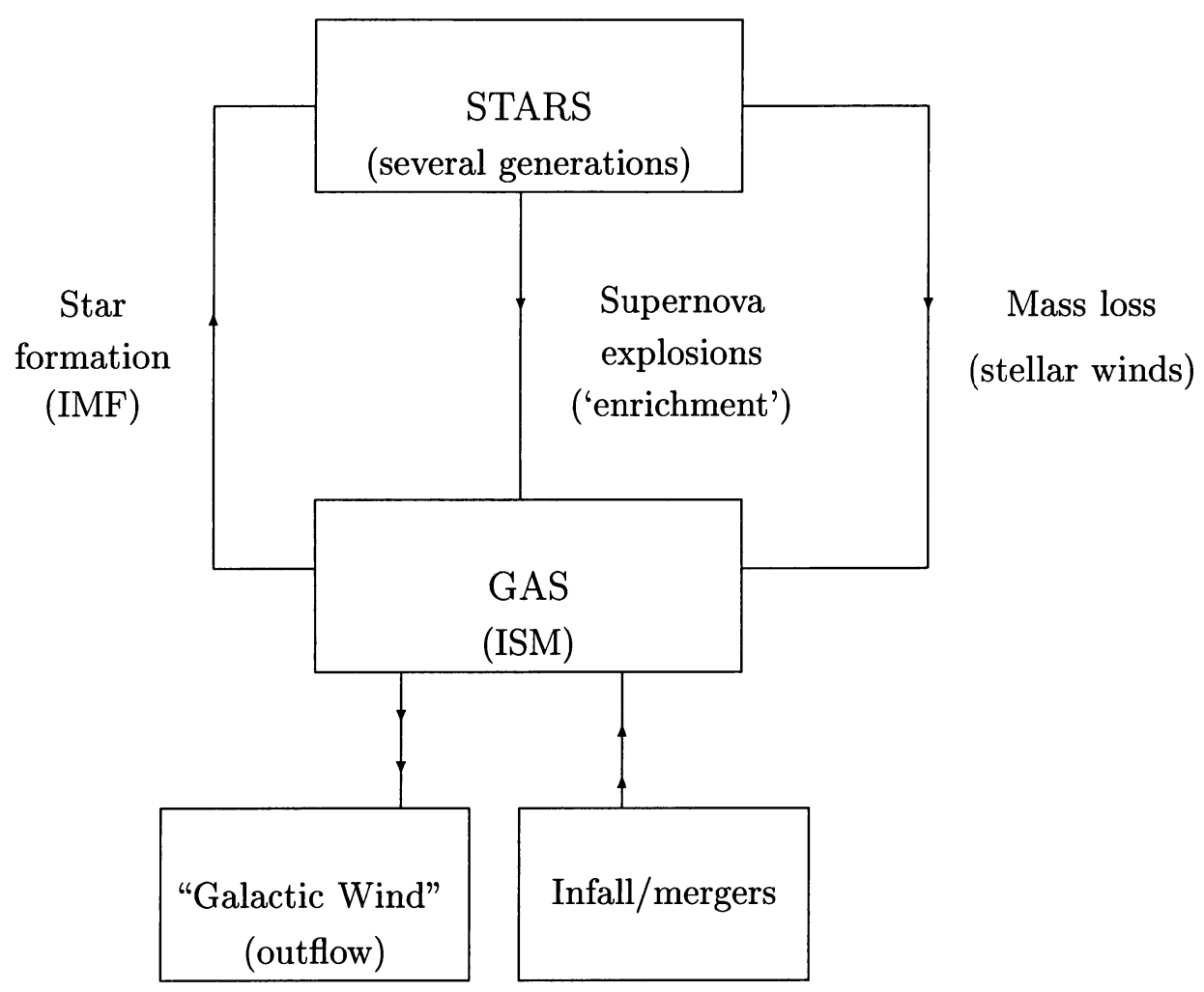

Figure 1. Chemical evolution of a galaxy

\section{The chemical evolution of a galaxy}

We can think of a galaxy as an ecosystem whose main components are stars and gas. As shown schematically in Figure 1, stars can lose mass to the interstellar medium (ISM) through mass loss in a stellar wind or, more spectacularly, in a supernova explosion. In either case, the material lost will chemically enrich the ISM. Gas in the ISM can in turn form new stars when conditions are suitable. If this is all that happens, then the galaxy is a closed system. Many galaxies may not be closed systems, however gas may be lost in an outflow (or 'galactic wind') if star formation becomes too vigorous; and new gas (and even stars) can be added by the infall or 
merger of smaller galaxies.

Models for the chemical evolution of galaxies (e.g. Arimoto \& Yoshii 1987; Matteucci 1992; Tantalo et al. 1996) attempt to reproduce the observed properties of nearby galaxies by simulating the collapse of a galaxy made of two components (luminous and dark matter) and allowing star formation to proceed in a well-determined way with consequent chemical enrichment of the ISM. They include the effects of galactic winds powered by supernova explosions and stellar winds from massive stars. Such models have been successful in reproducing many of the observed photometric and chemical properties of nearby galaxies, and suggest that the onset of a galactic wind is a key physical process in determining the chemical evolution of the most massive galaxies.

\subsection{STELLAR POPULATIONS ALONG THE HUBBLE SEQUENCE}

The outward appearance (Hubble type) of a galaxy depends on many factors, including kinematics, galaxy size, the relative amounts of gas and stars, the current star formation rate and the star formation history. In general, however, as we move along the Hubble sequence in the direction $\mathrm{E}-\mathrm{S} 0-\mathrm{Sa}-\mathrm{Sb}-\mathrm{Sc}-\mathrm{Sd}-\mathrm{Irr}$ we are moving from systems which have already turned most of their available gas into stars to systems which still have a relatively high gas fraction and active star formation. Hence we might expect that galaxies of similar Hubble type have also had a similar star formation and chemical enrichment history.

TABLE 1. Stellar populations along the Hubble sequence

\begin{tabular}{lll}
\hline Class & Dominant population & Closest Templates \\
\hline Dwarf & Varies/mixed & $\begin{array}{l}\text { LMC, SMC } \\
\text { Local Group dwarfs }\end{array}$ \\
Spiral & $\begin{array}{l}\text { Halo: old, metal-poor } \\
\text { Disk: mixed, young }\end{array}$ & Galaxy, M 31 \\
Elliptical & Old, metal-rich & Galactic bulge? \\
\hline
\end{tabular}

The problems I have chosen to discuss here relate mainly to giant elliptical galaxies. This is not because I think that giant ellipticals are the most interesting galaxies (although I do); but because disentangling the history of their chemical evolution is more difficult for these galaxies than for spiral and dwarf galaxies, where some members of the class are sufficiently nearby that we can resolve them into individual stars. 
The only giant elliptical galaxy in which individual stars have been resolved is NGC 5128, for which Soria et al. (1996) were able to detect the tip of the halo red giant branch with HST. O'Connell (1986) points out that HST easily resolves the brightest members of young stellar populations at the distance of the Virgo cluster, but that the brightest members of an old population with $[\mathrm{Fe} / \mathrm{H}]>0$ cannot be resolved outside the Local Group.

We are left with two possibilities. Either we try to disentangle the chemical evolution history of elliptical galaxies from their integrated light alone (either through spectra or broad-band colours), or we seek additional help from the only old and metal-rich population in which we CAN observe individual members - the stars of the Galactic bulge.

\subsection{MEASURING LINE-STRENGTH INDICES}

There are two problems in trying to disentangle the star formation history of a galaxy from the spectrum of its integrated light. Not only do we see the superposition of many spectra; but the velocity dispersion of the stars (due to their individual motions within the galaxy potential) limits the spectral resolution as well. For a giant elliptical galaxy, the internal velocity dispersion can be up to $300 \mathrm{~km} / \mathrm{s}$ (corresponding to line widths of several $\AA)$. Thus weak lines are blended or smeared out, and only strong lines can be measured. Since the $\mathrm{Mg}$ lines near $5200 \AA$ are among the strongest absorption lines, they have long been used as an abundance indicator for elliptical galaxies (e.g. Mould 1978; Gulati et al. 1991; Buzzoni et al. 1992; Barbuy 1994; Chavez et al. 1995).

The Lick line-strength indices (Faber et al. 1985) provide an easy way to quantify the absorption-line spectra of elliptical galaxies. The most commonly-used indices are $\mathrm{Mg}_{2}$ (covering the $\mathrm{Mg} b$ line and $\mathrm{MgH}$ molecular band), $<\mathrm{Fe}>$ (the mean of two Fe absorption lines) and $\mathrm{H} \beta$. Much effort has gone in to providing empirical abundance calibrations for the Lick indices, both for single stars and for composite populations (Burstein et al. 1984; Faber et al. 1985; Gorgas et al. 1993; Worthey et al. 1994). These measurements give us a broad, qualitative picture of the evolution of giant elliptical galaxies, as outlined below. Nevertheless, there remain some problems which will be discussed later.

\subsection{THE FORMATION OF ELLIPTICAL GALAXIES}

The first thing we can deduce from the integrated spectra of elliptical galaxies is that $\mathrm{K}$-giant stars dominate the light, and hence that the dominant stellar population is old. Since there appears to have been little or no recent star formation in these galaxies, it is plausible that the abundance distribu- 
tion (and especially the radial abundance gradient) provides a fossil record of the galaxy's formation.

Models in which elliptical galaxies formed by the dissipative collapse of a protogalaxy (Larson 1976; Carlberg 1985) predict that these galaxies should have steep radial abundance gradients (i.e. a decrease of 0.5 to 1.0 $\operatorname{dex}$ in $[\mathrm{Fe} / \mathrm{H}]$ over a decade in radius). Although abundance gradients are observed, however, (Faber 1977; Gorgas et al. 1990; Davies et al. 1993; Carollo \& Danziger 1994), they are much shallower (typically 0.2 dex per decade in radius) and uncorrelated with galaxy luminosity. This strongly favours mergers rather than dissipative collapse as the dominant formation mechanism for elliptical galaxies (White 1980), and the current view is that elliptical galaxies were 'assembled' from smaller subcomponents.

\subsection{POPULATION SYNTHESIS MODELS FOR THE INTEGRATED LIGHT}

O'Connell (1986) discusses two possible approaches to the spectral synthesis of an integrated galaxy spectrum. In both cases, the aim is to combine individual spectra (or spectral energy distributions) to produce a model composite. Recently, most workers have used the "evolutionary population synthesis" approach (Tinsley 1978; Bruzual \& Charlot 1993), in which they predict the spectral energy distribution (SED) resulting from a single-burst population and evolve this with time. A composite population can then be modelled by adding several single-burst models of different ages. The main variables are the stellar IMF and star formation rate, and it is clearly important to use a stellar library which contains all the stellar evolutionary stages which are present in the galaxy being modelled.

Charlot et al. (1996) compare several evolutionary synthesis models for elliptical galaxies, and discuss some of the limitations associated with them. The most serious of these are the treatment of stars in post-main sequence evolutionary stages; the poorly-determined temperature scale for the red giant branch and cool stars; and the lack of complete and accurate libraries of stellar spectra, especially for cool stars and non-solar metallicities. These problems relate directly to the topics discussed at this meeting.

\subsection{THE GALACTIC BULGE AS A TEMPLATE FOR ELLIPTICAL GALAXIES}

The Galactic bulge comprises the inner 1-2 kpc of our Galaxy. Its light is dominated by old, metal-rich stars (Arp 1965) with a wide abundance spread (Rich 1988). The mean abundance is slightly below solar (McWilliam \& Rich 1994; Sadler et al. 1996), with some stars possibly reaching 23 times solar abundance. Whitford (1978) first suggested that the stars of the Galactic bulge could be valuable templates for understanding the stellar 
populations of elliptical galaxies. The main arguments are that most of the bulge stars are old ( $>10 \mathrm{Gyr}$; Terndrup 1988), and that the abundances of the most metal-rich ones overlap the metallicity range deduced for elliptical galaxies.

The high (and patchy) reddening towards the Galactic bulge, along with the faintness of even the brightest bulge giants ( $\mathrm{V}=15-17$ mag.) and the problems of disk-star contamination and crowding make high-resolution spectroscopy difficult with $4 \mathrm{~m}$ class telescopes. In the near future, however, the new generation of 8-10 $\mathrm{m}$ telescopes will make it possible to carry out abundance analyses for large samples for metal-rich bulge giants (see Castro et al. 1996 for an early Keck spectrum of the bulge K-giant BW IV-167). These studies should both clarify the chemical evolution history of the bulge itself and provide improved stellar libraries for synthesis modelling of more distant galaxies.

\section{Problem 1: The UV upturn in elliptical galaxies}

Elliptical galaxies and the bulges of spirals show an upturn in their UV spectra at $\lambda<2500 \AA$, and their observed far-UV flux well exceeds that expected from the old stellar population which dominates the visible and IR light (e.g. Code \& Welch 1979; Burstein et al. 1988). In elliptical galaxies, the UV upturn increases with metallicity (Burstein et al. 1988), which cannot be explained by traditional stellar population models because increasing temperature ought to shift the red giant branch and asymptotic giant branch to cooler temperatures. The most plausible explanation appears to be that old, metal-rich stars can go through brief hot phases in their evolution (Greggio \& Renzini 1990).

Stellar evolution theory suggests several possible hot phases for old stars, including extreme horizontal branch (EHB) stars, AGB-manqué stars (or 'failed' AGB stars), and post-AGB (PAGB) stars. All of these are likely to be rare, because they represent short evolutionary phases, and only a few have been observed in globular clusters. The Galactic bulge promises to be a valuable laboratory for their study because it has so many stars that even short-lived phases can be observed in abundance. The main problem is to sift through large numbers of hot stars and separate the bulge stars from those in the foreground disk, so multi-object spectrographs (like the $2 \mathrm{dF}$ on the Anglo-Australian Telescope) are ideal for this work.

Figure 2 shows the expected location of the various hot-star candidates in the Galactic bulge CMD (RGB marks the bulge giant branch). Bertelli et al. (1996) have recently reported the likely signature of hot horizontalbranch stars in a bulge field near the Galactic Centre. 


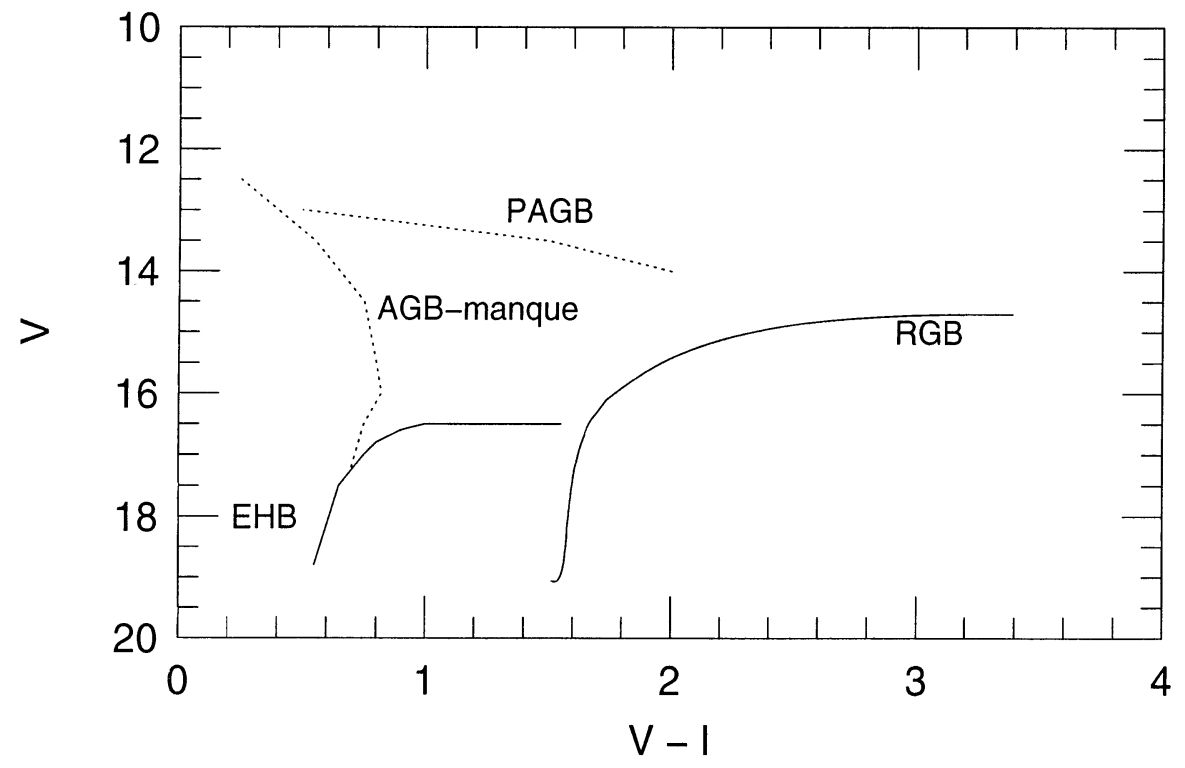

Figure 2. Schematic CMD for candidate UV-bright stars in the Galactic bulge

\section{Problem 2: Enhanced $\mathrm{Mg} / \mathrm{Fe}$ in ellipticals, and the fate of iron from $\mathrm{SNeI}$}

From the relative behaviour of the $\mathrm{Mg}_{2}$ and $<\mathrm{Fe}>$ indices, Worthey et al. (1992) suggest that magnesium is overabundant relative to iron in elliptical galaxies, with $[\mathrm{Mg} / \mathrm{Fe}]=+0.2$ to 0.3 (McWilliam \& Rich (1994) derive a similar enhancement for stars in the Galactic bulge). Enhanced $\mathrm{Mg}$ relative to Fe could plausibly arise if most of the stars in elliptical galaxies formed very quickly (i.e. in $<1 \mathrm{Gyr}$ ), with very little star formation thereafter. In this case, there would be rapid chemical enrichment from type II supernovae, with a corresponding enhancement of $\alpha$-elements such as magnesium.

While this explains what we observe in the integrated stellar light, nucleosynthesis doesn't stop when star formation stops. As noted in Figure 1 , the interstellar medium (ISM) will continue to be enriched by stellar mass loss and by Type Ia supernovae, which should contribute substantial amounts of iron. Surprisingly, though, the ASCA satellite measured iron abundances in the hot ISM of giant elliptical galaxies which are LOWER than those in the stellar galaxy (e.g. Awaki et al. 1994). This poses two problems: (i) the hot ISM is believed to be shed by stars, yet it has a lower iron abundance than the stars from which it arose; and (ii) if the iron from type Ia supernovae has not been recycled into new generations of stars, and 
is not in the hot ISM, where could it have gone?

A recent paper by Arimoto et al. (1997) discusses the iron discrepancy revealed by the ASCA data, and explores possible ways of hiding or diluting iron in the ISM of ellipticals. None of these appears plausible, and the only alternatives appear to be that either the diagnostic tools currently used to infer abundances from X-ray spectra give systematically low values in certain conditions (which the authors believe is plausible), or we need to rethink our models for the chemical evolution of galaxies.

\section{Problem 3: Dating star formation - the age-metallicity de- generacy}

A major problem in disentangling the star formation history of ellipticals is that an 'age-metallicity degeneracy' affects broad-band colours and most spectral lines (e.g. Renzini 1986; Worthey et al. 1996). Thus changes in the colour or line-strength of an elliptical galaxy can generally be attributed almost equally plausibly to either an age or a metallicity change (a possible exception is the $\mathrm{H} \beta$ index, which is more sensitive to temperature than to abundance). For this reason, the epoch at which most elliptical galaxies formed most of their stars remains uncertain. Some of the arguments for 'old' and 'young' ages are:

Arguments that the bulk of the stellar population is old (>12 Gyr), from Renzini (1995):

- Clusters of galaxies at redshifts z 1 contain bright, red galaxies whose colours are consistent with passive evolution since $\mathrm{z}>2$.

- The tightness of the fundamental plane for nearby galaxies implies either a very small age dispersion or formation at $z>2$.

- QSOs at $z>3$ show strong metal enrichment, suggesting that abundances in the central regions were already high 15 Gyr ago.

- If smaller ellipticals are much younger than bigger ones, this is hard to reconcile with a picture in which (as discussed earlier) larger ellipticals are "assembled" from smaller ones.

Arguments that the mean stellar ages of E nuclei are young (3-12 Gyr), from Faber et al. (1995):

- The $\mathrm{H} \beta$ line can be used as a temperature (and hence age) indicator, independent of metallicity.

- In the nuclei of elliptical galaxies, $\mathrm{H} \beta$ is stronger than expected from single-burst models of an old population.

- The flat $\mathrm{H} \beta$ gradients seen in most elliptical galaxies imply that the outer parts of these galaxies are both older and more metal-poor than the central regions. 
A possible reconciliation of these views is offered by Bressan et al. (1996), who point out that the strength of the UV upturn (1550-V colour) may be a powerful age indicator for elliptical galaxies. They suggest that all ellipticals have old (13-15 Gyr) populations, but that their star formation histories differ, with star formation continuing for much longer in some galaxies than others.

We now have good-quality spectra of galaxies and increasingly sophisticated models, but it is unfortunate that the empirical calibration of the line-strength indices for metal-rich stars still rests on a handful of solar neighbourhood stars.

\section{Summary}

I have given a brief introduction to a few problems in extragalactic astronomy which have direct links to the topic of this meeting. We could make progress with several of these if we understood more about the late evolution of stars of solar metallicity and above. The message I would like to deliver is a simple one: studies of the formation and evolution of galaxies would be on firmer ground if the old, metal-rich stellar population of the Galactic bulge were as well observed, and as well understood, as the stellar populations of the Galactic bulge and halo.

I thank Don Terndrup for providing the diagram used in Figure 2.

\section{References}

Arimoto, N. and Yoshii, Y., 1987. $A \& A, \mathbf{1 7 3}, 23$.

Arimoto, N., Matsushita, K., Ishimaru, Y., Ohashi, T. and Renzini, A., 1997. ApJ, 477, 128.

Arp, H., 1965. ApJ, 141, 43.

Awaki, H., et al., 1994. PASJ, 46, L65.

Barbuy, B., 1994. AJ, 430, 218.

Bertelli, G., Bressan, A., Chiosi, C. and Ng, Y.K., 1996. $A \& A$, 310, 115.

Bressan, A., Chiosi, C. and Tantalo, R., 1996. $A \& A$, 311, 425.

Buzzoni, A., Gariboldo, G. and Mantegazza, L., 1992. $A J$, 103, 1814.

Bruzual, A.G. and Charlot, S., 1993. ApJ, 405, 538.

Burstein, D., Faber, S.M., Gaskell, C.M. and Krumm, N., 1984. ApJ, 287, 586.

Burstein, D., Bertola, F., Buson, L., Faber, S.M. and Lauer, T.R., 1988. ApJ, 328, 440.

Carlberg, R.. 1984. ApJ, 286, 404.

Carollo, C.M. and Danziger, I.J., 1994. MNRAS, 270, 523.

Castro, S., Rich, R.M., McWilliam, A., Ho, L.C., Spinrad, H., Filippenko, A.V., and Bell, R.A., 1996. $A J, \mathbf{1 1 1}, 2439$.

Chavez, M., Malagnini, M.L. and Morossi, C., 1995. ApJ, 440, 210.

Charlot, S., Worthey, G. and Bressan, A., 1996. ApJ, 457, 625.

Code, A.D. and Welch, G.A., 1979. ApJ, 228, 95.

Davies, R.L., Sadler, E.M. and Peletier, R., 1993. MNRAS, 262, 650.

Faber, S.M., 1977. In The Evolution of Galaxies and Stellar Populations, Yale University Press, p. 157. 
Faber, S.M., Friel, E.D., Burstein, D. and Gaskell, C.M., 1985. ApJS, 57, 711.

Faber, S.M., Trager, S.C., Gonzalez, J.J. and Worthey, G., 1995. In Stellar populations, IAU Symposium 164, p. 249.

Gorgas, J., Efstathiou, G. and Aragon Salamanca, A., 1990. MNRAS, 245, 217.

Gorgas, J., Faber, S.M., Burstein, D., Gonzalez, J.J., Courteau, S. and Prosser, C., 1993. ApJS, 86, 153.

Greggio, L. and Renzini, A., 1990. ApJ, 364, 35.

Gulati, R.K., Malagnini, M.L. and Morossi, C., 1991. A\&A, 247, 447.

Larson, R.B., 1976. MNRAS, 176, 31.

Matteucci, F., 1991. ApJ, 397, 32.

McWilliam, A. and Rich, R.M., 1994. ApJS, 91, 749.

Mould, J.R., 1978. ApJ, 220, 434.

O'Connell, R.W., 1986. In Stellar populations, Cambridge University Press , p. 167.

O'Connell, R.W., 1995. In Stellar populations, IAU Symposium 164, p. 301.

Renzini, A., 1986. In Stellar populations, Cambridge University Press , p. 213.

Renzini, A., 1995. In Stellar populations, IAU Symposium 164, p. 325.

Rich, R.M., 1988. AJ, 95, 828.

Sadler, E.M., Rich, R.M. and Terndrup, D.M., 1996. $A J, \mathbf{1 1 2}, 171$.

Soria, R., et al., 1996. ApJ, 465, 79.

Steidel, C.C., Giavalisco, M., Pettini, M., Dickinson, M. and Adelberger, K.L., 1996. ApJ, 462, L17.

Tantalo, R., Chiosi, C., Bressan, A. and Fagotto, F., 1996. $A \& A$, 311, 361.

Terndrup, D.M., 1988. $A J, \mathbf{9 6}, 884$.

Tinsley, B.M., 1978. ApJ, 222, 14.

White, S.D.M., 1980. MNRAS, 191, 1P.

Whitford, A.E., 1978. ApJ, 226, 777.

Worthey, G., Faber, S.M. and Gonzalez, J.J., 1992. ApJ, 398, 69.

Worthey, G., Faber, S.M., Gonzalez, J.J. and Burstein, D. 1994. ApJS, 94, 687.

Worthey, G., Trager, S.C. and Faber, S.M., 1996. In Fresh Views of Elliptical Galaxies, ASP Conf. Series, 86, 203.

\section{DISCUSSION}

ROGER BELL: Houdashelt and I have calculated galaxy spectra, using synthetic spectra and isochrones. We can calculate Lick-type indices from these spectra without making use of the fitting functions. Do you know if there are galaxy spectra, rather than indices, which we could compare with to study the possible $[\mathrm{Mg} / \mathrm{Fe}]$ versus $[\mathrm{Fe} / \mathrm{H}]$ correlation?

ELAINE SADLER: I agree that the approach you describe is an excellent one, though at present I would worry a little about the synthetic spectra for the coolest stars. The Lick indices are attractive to observers because they are robust - for example, they don't require that the galaxy spectra be flux-calibrated, so data from non-photometric nights can still be used. Nevertheless, libraries of good-quality, flux-calibrated spectra do exist for nearby galaxies (e.g. Kennicutt 1992; ApJS 79, 255). 